\title{
AUTHOR INDEX Volume 15 (2010)
}

Abboud, R. J., see Jariwala, A. C.

Al-Jamali, J., Gerlach, U. V. \& Zajonc, H., Benign Fibrous Histocytoma of the Distal Radius: A Report of a Case and a Review of the Literature

Allom, R., see Colegate-Stone, T.

Amirfeyz, R., see Atwal, N. S.

Amirfeyz, R., Mehendale, S., Tyrrell, S., Bhatia, R., Leslie, I. \& Bannister, G., Katz and Stirrat Hand Diagram Revisited

Anastasopoulos, N., see Natsis, K.

Andreyechen, M., see Pollock, J.

Aoki, M., see Iba, K.

Arnold, G. P., see Jariwala, A. C.

Atwal, N. S., Clark, D. A., Amirfeyz, R. \& Bhatia, R., Salvage of a Failed Sauvé-Kapandji Procedure Using a Total Distal Radio-Ulnar Joint Replacement

Avakian, Z., see Edmunds, I.

Bannister, G., see Amirfeyz, R.

Baraza, N., see Hull, P.

Bhatia, R., see Atwal, N. S.

Bhatia, R., see Amirfeyz, R.

Brewster, M., see Hull, P.

Calandruccio, J. H., see Duncan, S. F. M.

Chan, J. K. K., Choa, R. M., Chung, D., Sleat, G., Warwick, R. \& Smith, G. D., High Resolution Ultrasonography of the Hand and Wrist: Three-Year Experience at a District General Hospital Trust

Charalambous, C. P., see Ravenscroft, M. J.

Charalambous, C. P., see Tryfonidis, M.

Charalambous, C. P., Mills, S. P. \& Hayton, M. J., Gradual Distraction Using An External Fixator Followed by Open Reduction in the Treatment of Chronic Lunate Dislocation

Cheung, J. P. Y., Fung, B. K.-K. \& Ip, W.-Y., Mycobacterium marinum Infection of the Deep Structures of the Hand and Wrist: 25 Years of Experience

Choa, R. M., see Chan, J. K. K.

Chung, D., see Chan, J. K. K.

Clark, D. A., see Atwal, N. S.

Colegate-Stone, T., Allom, R., Tavakkolizadeh, A. \& Compson, J., An Analysis of Partial Width Flexor Tendon Graft Preparation on Porcine Models: The Optimal Split?

Compson, J., see Colegate-Stone, T.

Conway, A. F., see Pollock, J.

Cooper, J. P., see Ya'ish, F.

Costa, M., see Hull, P.

Craigen, M. A., see Ya'ish, F.
3 (2010) $237-241$

$2(2010) 127-129$

2 (2010) 99-102

2 (2010) 119-122

2 (2010) 71-73

3 (2010) 235-236

3 (2010) 193-201

1 (2010) 11-15

3 (2010) 237-241

2 (2010) 119-122

2 (2010) 109-113

2 (2010) 71-73

3 (2010) 185-191

2 (2010) 119-122

2 (2010) 71-73

3 (2010) 185-191

2 (2010) 75-80

3 (2010) 177-183

2 (2010) 95-98

3 (2010) 161-164

1 (2010) 27-29

3 (2010) 211-216

3 (2010) 177-183

3 (2010) 177-183

2 (2010) 119-122

2 (2010) 99-102

2 (2010) 99-102

3 (2010) 193-201

3 (2010) 173-176

3 (2010) 185-191

3 (2010) 173-176 
Crockarell, J. R., see Duncan, S. F. M.

DiPrimio, G., see Pollock, J.

Duncan, S. F. M., Calandruccio, J. H., Merritt, M. V., Crockarell, J. R. \& Kakinoki, R., A Comparison of Workers' Compensation Patients and Nonworkers' Compensation Patients Undergoing Endoscopic Carpal Tunnel Release

Edmunds, I. \& Avakian, Z., Hand Surgery on Anticoagulated Patients: A Prospective Study of 121 Operations Fujioka, H., see Nagura, I.

Fujioka, H., see Kanatani, T.

Fujitani, R., see Ono, H.

Funakoshi, T., see Iwasaki, N.

Fung, B. K.-K., see Cheung, J. P. Y.

Furue, Y., see Muraoka, S.

Furuta, K., see Ono, H.

Gerlach, U. V., see Al-Jamali, J.

Giachino, A. A., see Pollock, J.

Goodson, A., Morgan, M., Rajeswaran, G., Lee, J. \& Katsarma, E., Current Management of Jersey Finger in Rugby Players: Case Series and Literature Review

Goshtasby, P. H., Wheeler, D. R. \& Moy, 0. J., Risk Factors for Trigger Finger Occurrence After Carpal Tunnel Release

Goto, A., Kunihiro, 0., Murase, T. \& Moritomo, H., The Dorsal Cutaneous Branch of the Ulnar Nerve: An Anatomical Study

Grant, I., see Shah, A. K.

Guzzetti, T. \& Thione, A., Delayed Successful Free Fibula Flap for Treatment of Complex Arm Gunshot Wound - A Case Report

Haflah, N. H. M., Rashid, A. H. A. \& Sapuan, J., Partial Anterior Interosseous Nerve Palsy: Isolated Neuropraxia of the Branch to Flexor Pollicis Longus

Hamanaka, I., see Yoshida, A.

Hara, A., see Narushima, Y.

Hayton, M. J., see Charalambous, C. P.

Hayton, M. J., see Tryfonidis, M.

Hoshino, Y., Saito, N. \& Kuroda, H., Surgical Treatment of Mucous Cysts on Fingers without Skin Excision

Hrushowy, H., see Pollock, J.

Hull, P., Baraza, N., Whalley, H., Brewster, M. \& Costa, M., Dorsally Displaced Fractures of the Distal Radius - A Study of Preferred Treatment Options Among UK Trauma and Orthopaedic Surgeons

Iba, K., Wada, T., Tamakawa, M., Aoki, M. \& Yamashita, T., Diffusion-Weighted Magnetic Resonance Imaging of the Ulnar Nerve in Cubital Tunnel Syndrome

Ikehata, T., see Komura, S.

Ip, W.-Y., see Cheung, J. P. Y.

Ito, H., see Kitagawa, Y.

Itoh, S., Miura, T., Oka, H., Nakagawa, T. \& Nakamura, K., Reproducibility of Measurements of Thumb Abduction

Iwasaki, N., see Matsuhashi, T.

Iwasaki, N., Masuko, T., Funakoshi, T. \& Minami, A., Elderly Kendo (Japanese Fencing) Player with Kienböck's Disease in One Wrist and Preiser's Disease in the Other Wrist: A Case Report

Jacob, S., see Tryfonidis, M.

Jariwala, A. C., Scott, I., Arnold, G. P., Abboud, R. J. \& Wigderowitz, C. A., Objective Assessment of the Alterations in Wrist Mobility with the Fastrak ${ }^{\circledR}$ System Following Dorsal Capsulodesis

Jass, G. K., see Tryfonidis, M.

Jianmongkol, S., see Tiyaworanan, P.

Kakinoki, R., see Duncan, S. F. M.

Kanatani, T., Yamasaki, K. \& Fujioka, H., Carpal Tunnel Syndrome Associated with a Fracture of a Silicone Implant for Kienböck's Disease: Two Case Reports
2 (2010) $75-80$

3 (2010) 193-201

2 (2010) 75-80

2 (2010) 109-113

2 (2010) 123-125

3 (2010) 225-227

2 (2010) 139-144

1 (2010) 47-51

3 (2010) 211-216

1 (2010) 61-64

2 (2010) 139-144

2 (2010) 127-129

3 (2010) 193-201

2 (2010) 103-107

2 (2010) 81-87

3 (2010) 165-168

1 (2010) 31-33

1 (2010) 35-39

3 (2010) 221-223

2 (2010) 65-69

1 (2010) 57-60

1 (2010) 27-29

3 (2010) 161-164

2 (2010) 145-148

3 (2010) 193-201

3 (2010) 185-191

1 (2010) 11-15

3 (2010) 233-234

3 (2010) 211-216

2 (2010) 115-117

1 (2010) 7-10

1 (2010) 53-55

1 (2010) 47-51

3 (2010) 161-164

3 (2010) 237-241

3 (2010) 161-164

3 (2010) 157-159

2 (2010) 75-80

3 (2010) 225-227 
Kanaya, K., Wada, T. \& Yamashita, T., Scaphoid Dislocation Associated with Axial Carpal Dissociation During Volar Flexion of the Wrist: A Case Report

Kapickis, M., Lahiri, A. \& Lim, A. Y.-T., Different Angiogenic Behaviour of Ligated Saphenous and Epigastric Vascular Bundles in Avascular Environment: An In-Vivo Experimental Study

Kato, H., see Uehara, M.

Katsarma, E., see Goodson, A.

Kawashima, M., see Muraoka, S.

Keefer, E. P., see Meftah, M.

Keskin, M., see Seyhan, N.

Kitagawa, Y., Sawaizumi, T. \& Ito, H., Myositis Ossificans of the Supinator Muscle Causing Posterior Interosseous Nerve Palsy: A Case Report

Komura, S., Suzuki, Y. \& Ikehata, T., Simultaneous Fracture of the Waist of the Scaphoid and the Hook of the Hamate

Kosiyatrakul, A., see Luenam, S.

Kunihiro, 0., see Goto, A.

Kuroda, H., see Hoshino, Y.

Kusunose, K., see Narushima, Y.

Lahiri, A., see Kapickis, M.

Lee, J., see Goodson, A.

Leslie, I., see Amirfeyz, R.

Lim, A. Y.-T., see Kapickis, M.

Luenam, S., Kosiyatrakul, A. \& Prachaporn, S., Triggering of Extensor Pollicis Longus Tendon: Case Report

Masuko, T., see Iwasaki, N.

Matsuhashi, T., Iwasaki, N., Nishida, K., Motomiya, M. \& Minami, A., Palmar Subluxation of the Thumb Carpometacarpal Joint Following the Median Nerve Recurrent Branch Injury

Meftah, M., Keefer, E. P., Panagopoulos, G. \& Steven Yang, S., Arthroscopic Wafer Resection for Ulnar Impaction Syndrome: Prediction of Outcomes

Mehendale, S., see Amirfeyz, R.

Mennen, U., see van der Westhuizen, J.

Merritt, M. V., see Duncan, S. F. M.

Mills, S. P., see Tryfonidis, M.

Mills, S. P., see Charalambous, C. P.

Mills, S. P., see Ravenscroft, M. J.

Minami, A., see Iwasaki, N.

Minami, A., see Matsuhashi, T.

Miura, T., see Itoh, S.

Morgan, M., see Goodson, A.

Moritomo, H., see Goto, A.

Motomiya, M., see Matsuhashi, T.

Moy, 0. J., see Goshtasby, P. H.

Muraoka, S., Furue, Y. \& Kawashima, M., Irreducible Open Dorsal Dislocation of the Proximal Interphalangeal Joint: A Case Report

Murase, T., see Goto, A.

Nabeshima, Y., see Nagura, I.

Nagura, I., Fujioka, H. \& Nabeshima, Y., Simultaneous Fractures of the Scaphoid, Proximal and Distal End of the Radius: A Case Report

Nakagawa, T., see Itoh, S.

Nakamura, K., see Itoh, S.

Narushima, Y., Hara, A. \& Kusunose, K., An Unusual Fracture-Dislocation of the Trapeziometacarpal Joint of the Thumb: A Case Report

Natsis, K., Papathanasiou, E. \& Anastasopoulos, N., Accessory Extensor Digiti Minimi Muscle Simulating a Soft Tissue Mass During Surgery: A Case Report

3 (2010) 229-232

1 (2010) 17-25

3 (2010) 217-220

2 (2010) 103-107

1 (2010) 61-64

2 (2010) 89-93

2 (2010) 131-133

2 (2010) 115-117

3 (2010) 233-234

2 (2010) 135-137

3 (2010) 165-168

2 (2010) 145-148

1 (2010) 57-60

1 (2010) 17-25

2 (2010) 103-107

2 (2010) 71-73

1 (2010) 17-25

2 (2010) 135-137

1 (2010) 47-51

1 (2010) 53-55

2 (2010) 89-93

2 (2010) 71-73

3 (2010) 203-210

2 (2010) 75-80

3 (2010) 161-164

1 (2010) 27-29

2 (2010) 95-98

1 (2010) 47-51

1 (2010) 53-55

1 (2010) 7-10

2 (2010) 103-107

3 (2010) 165-168

1 (2010) 53-55

2 (2010) 81-87

1 (2010) 61-64

3 (2010) 165-168

2 (2010) 123-125

2 (2010) 123-125

1 (2010) 7-10

1 (2010) 7-10

1 (2010) 57-60

3 (2010) 235-236 
Nishida, K., see Matsuhashi, T.

Oka, H., see Itoh, S.

Okutsu, I., see Yoshida, A.

Okutsu, I., How I Developed the World's First Evidence-Based Endoscopic Management of Carpal Tunnel Syndrome

Ono, H., Furuta, K. \& Fujitani, R., Radiolunate Fusion Using Vascularized Radius Bridging Graft for the Treatment of Advanced Kienböck Disease: Two Cases Report

Panagopoulos, G., see Meftah, M.

Papathanasiou, E., see Natsis, K.

Pollock, J., Giachino, A. A., Rakhra, K., DiPrimio, G., Hrushowy, H., Conway, A. F. \& Andreyechen, M., SLAC Wrist in the Absence of Recognised Trauma and CPPD

Prachaporn, S., see Luenam, S.

Pretell-Mazzini, J., see Rodriguez-Martin, J.

Rajeswaran, G., see Goodson, A.

Rakhra, K., see Pollock, J.

Rashid, A. H. A., see Haflah, N. H. M.

Ravenscroft, M. J., Charalambous, C. P., Mills, S. P., Woodruff, M. J. \& Stanley, J. K., Bone-Cement Interface Strength in Distal Radii Using Two Medullary Canal Preparation Techniques: Carbon Dioxide Jet Cleaning Versus Syringed Saline

Rodriguez-Martin, J., Pretell-Mazzini, J. \& Vidal-Bujanda, C., Unusual Pattern of Essex-Lopresti Injury with Negative Plain Radiographs of the Wrist: A Case Report and Literature Review

Saito, N., see Hoshino, Y.

Saito, T., see Takigami, H.

Sakano, H., see Takigami, H.

Sapuan, J., see Haflah, N. H. M.

Savaci, N., see Seyhan, N.

Sawaizumi, T., see Kitagawa, Y.

Scott, I., see Jariwala, A. C.

Seyhan, N., Keskin, M., Tosun, Z. \& Savaci, N., Extensor Tendon Rupture Due to Cutaneous Leichmaniasis: A Case Report

Shah, A. K. \& Grant, I., Intravascular Papillary Endothelial Hyperplasia (Masson's Tumour) Causing a Soft-Tissue Mass in the Upper Limb

Sleat, G., see Chan, J. K. K.

Smith, G. D., see Chan, J. K. K.

Sreedharan, S., Yam, A. K. T. \& Tay, S. C., Self-Reported Outcome Following Anterior Transposition of Ulnar Nerve in the Elderly

Stanley, J. K., see Ravenscroft, M. J.

Stanley, J. K., see Tryfonidis, M.

Steven Yang, S., see Meftah, M.

Suzuki, Y., see Komura, S.

Takigami, H., Sakano, H. \& Saito, T., Internal Fixation with the Low Profile Plate System Compared with Kirschner Wire Fixation: Clinical Results of Treatment for Metacarpal and Phalangeal Fractures

Tamakawa, M., see Iba, K.

Tavakkolizadeh, A., see Colegate-Stone, T.

Tay, S. C., see Sreedharan, S.

Thammaroj, T., see Tiyaworanan, P.

Thione, A., see Guzzetti, T.

Tiyaworanan, P., Jianmongkol, S. \& Thammaroj, T., Anatomical Study of Arcade of Struthers

Tosun, Z., see Seyhan, N.

Tryfonidis, M., Charalambous, C. P., Mills, S. P., Jass, G. K., Jacob, S., Stanley, J. K. \& Hayton, M. J., Distal Radial and Ulnar Landmarks Used in Percutaneous Pin Fixation: Anatomical Relationship to the Superficial Radial and Ulnar Nerves
1 (2010) 53-55

1 (2010) 7-10

2 (2010) 65-69

3 (2010) 149-155

2 (2010) 139-144

2 (2010) 89-93

3 (2010) 235-236

3 (2010) 193-201

2 (2010) 135-137

1 (2010) 41-45

2 (2010) 103-107

3 (2010) 193-201

3 (2010) 221-223

2 (2010) 95-98

1 (2010) 41-45

2 (2010) 145-148

1 (2010) $1-6$

1 (2010) 1-6

3 (2010) 221-223

2 (2010) 131-133

2 (2010) 115-117

3 (2010) 237-241

2 (2010) 131-133

1 (2010) 31-33

3 (2010) 177-183

3 (2010) 177-183

3 (2010) 169-172

2 (2010) 95-98

3 (2010) 161-164

2 (2010) 89-93

3 (2010) 233-234

1 (2010) 1-6

1 (2010) 11-15

2 (2010) 99-102

3 (2010) 169-172

3 (2010) 157-159

1 (2010) 35-39

3 (2010) 157-159

2 (2010) 131-133

3 (2010) 161-164 
Tyrrell, S., see Amirfeyz, R.

$2(2010) 71-73$

Uehara, M., Yamazaki, H. \& Kato, H., Acute Plastic Bowing of the Radius with a Distal Radioulnar Joint Injury:

A Case Report

van der Westhuizen, J. \& Mennen, U., A Working Classification for the Management

of Scapho-Trapezium-Trapezoid Osteo-Arthritis

Vidal-Bujanda, C., see Rodriguez-Martin, J.

Wada, T., see Iba, K.

Wada, T., see Kanaya, K.

Warwick, R., see Chan, J. K. K.

Whalley, H., see Hull, P.

Wheeler, D. R., see Goshtasby, P. H.

Wigderowitz, C. A., see Jariwala, A. C.

Woodruff, M. J., see Ravenscroft, M. J.

Ya'ish, F., Cooper, J. P. \& Craigen, M. A., "Mood Rings": A New Method of Objective Clinical Assessment of Peripheral Nerve Injuries

Yamasaki, K., see Kanatani, T.

Yamashita, T., see Kanaya, K.

Yamashita, T., see Iba, K.

Yamazaki, H., see Uehara, M.

Yam, A. K. T., see Sreedharan, S.

Yoshida, A., Okutsu, I. \& Hamanaka, I., A New Diagnostic Provocation Test for Carpal Tunnel Syndrome: Okutsu Test

Zajonc, H., see Al-Jamali, J.

3 (2010) 217-220

3 (2010) 203-210

1 (2010) 41-45

1 (2010) $11-15$

3 (2010) 229-232

3 (2010) $177-183$

3 (2010) 185-191

2 (2010) 81-87

3 (2010) 237-241

2 (2010) 95-98

3 (2010) $173-176$

3 (2010) 225-227

3 (2010) 229-232

1 (2010) $11-15$

3 (2010) 217-220

3 (2010) 169-172

2 (2010) 65-69

2 (2010) $127-129$ 